\title{
The Effect of He-Ne and Diode Lasers on the Electrical Characteristics of Silicon Diode
}

\author{
Yousif Hassan Alsheikh Abd Alraheim, Zainab Abdulla Mohamed Taha, Zainab Mohamed Taha
}

Department of Applied Physics \& Mathematics, Omdurman Ahlia University, Omdurman, Khartoum, Sudan

\author{
Email address: \\ yousif.alsheikh@yahoo.com (Y. H. A. A. Alraheim) \\ ${ }^{*}$ Corresponding author
}

\section{To cite this article:}

Yousif Hassan Alsheikh Abd Alraheim, Zainab Abdulla Mohamed Taha, Zainab Mohamed Taha. The Effect of He-Ne and Diode Lasers on the Electrical Characteristics of Silicon Diode. American Journal of Optics and Photonics. Vol. 6, No. 1, 2018, pp. 8-13. doi: 10.11648/j.ajop.20180601.12

Received: October 30, 2017; Accepted: November 11, 2017; Published: January 18, 2018

\begin{abstract}
The effect of He-Ne $(632.8 \mathrm{~nm})$ and diode $(650 \mathrm{~nm})$ lasers on the electrical characteristics of silicon diode have been studied. The electrical characteristics of the diode were recorded before laser irradiations, then the diode is subjected to $\mathrm{He}-\mathrm{Ne}$ laser for 5 and 10 minutes and then the diode electrical characteristics were recorded for each time of exposure and the same was done in the case of irradiation with diode laser. The electrical characteristics of the diode before and after laser irradiations were compared and thermal effect was noticed when compared the effect of lasers irradiation and the well known temperature effect on the electrical characteristics of the diode. It was found that the effect of the He-Ne $(632.8 \mathrm{~nm})$ and diode laser $(650 \mathrm{~nm})$ on the electrical characteristics of silicon diode at exposure time of 5 minutes were comparable but for 10 minutes of exposure the effect of He-Ne laser irradiation on the characteristics was different from that of diode laser and this is due to the fact that the two lasers has different properties.
\end{abstract}

Keywords: He-Ne Laser, Diode Laser, Electrical Characteristics of Silicon Diode, Laser Modes of Interaction

\section{Introduction}

The word laser is an acronym of the words: Light Amplification by Stimulated Emission of Radiation [1]. It is used to describe the device that produce that light with that unusual properties or to describe the light itself, and in some manner to describe the process of producing that light (lasing). The applications of lasers depend on the unusual properties of laser light, ones that are different from the properties of light from conventional sources [2]. A p-n junction diode can be fabricated by doping the semiconductor material with opposite doping impurities (i.e., acceptor or donor impurities) to form the $\mathrm{p}$ and $\mathrm{n}$ regions of the diode. The basic device theory used in predicting the currentvoltage $(\mathrm{I}-\mathrm{V})$ characteristics in a p-n junction diode was first developed by Shockley [3].

Shockley diode equation is given by:

$$
J=J_{0}\left(e^{\frac{q V}{K T}}-1\right)
$$

Where $\mathrm{J}$ is the diode current density and $\mathrm{J}_{0}$ is the saturation current density, $\mathrm{q}$ is the electron charge, $\mathrm{V}$ is the diode voltage, $\mathrm{K}$ is the Boltzmann's constant and $\mathrm{T}$ represents the temperature. It is well known that when the temperature raises the forward bias region of the I-V curve of the diode is shifted to the right. The $\mathrm{p}-\mathrm{n}$ junction is the basis of many other electronic devices [4]. Diode has many types that include normal diode, light emitting diode etc., the principle of emitting light from light emitting diode is same as that used to produce laser light from the diode laser but the emission of light from the diode laser is due to stimulated emission in contrast to spontaneous emission in the case of light emitting diode. Diode lasers operate in many wavelengths and the emitted wavelength depends on the band gab energy of the p-n junction. Diode lasers like all other laser machines have many applications.

Diode lasers find applications in optical communication systems as a light source with good properties instead of light emitting diodes.

The industrial laser machine tool is different from traditional one in that it is a flexible machine tool. It produces 
a beam of light with unique properties. Its light can be controlled accurately: it can be focused to a small spot, providing an intense source of energy that is ideal for penetrating materials; or spread into a diffuse heating pattern to treat surfaces. As well as processing materials by thermal modes, the interaction between the photons of the laser beam and atoms in materials enables processes to be performed athermally (without heat): bonds can be made and broken. The beam can be manipulated with optical components to perform a variety of operations simultaneously, or switched between locations for sequential processing $[5,6]$.

Examples of laser applications that are achieved from thermal and/or athermal modes of laser interaction with materials are: In the applications in which one generally tries to avoid vaporization and material removal such as applications of laser welding and applications of laser for surface treatments those applications are based on laserinduced heating and melting and therefore thermal mode is dominating for such applications [2].

Also for laser initiated chemical reaction traditional thermal energy were used and laser when used in this applications the rate of chemical reaction was shown to be high in addition to other advantages such as selective absorption the vibrational states in reactant molecules, etc.

Clearly laser spectroscopic techniques such as laser induced breakdown spectroscopy and laser absorption spectroscopy are examples of an applications of the lasers that are classified as athermal mode. Some applications that require material removal such as hole drilling, cutting, and marking may depends on both thermal and athermal modes of interaction.

The electrical characteristics of diodes together with thermal characteristics of the diodes and other figure of merits such as efficiency are the key feature of the diode to be used in particular applications [7].

In this work we studied the effect of laser irradiations on the electrical characteristics of silicon diode. He-Ne $(632.8 \mathrm{~nm})$ and Diode $(650 \mathrm{~nm})$ lasers were used to accomplish the study.

\section{Materials, Tools and Methods}

\subsection{Materials}

The materials used are a number of silicon diode. The diode is an electronic device that allow current to pass in single direction only. Silicon diode used here was imported from china and it was available at the Sudanese electronic equipment's and components companies at Khartoum state.

\subsection{Tools}

Many tools and devices used to accomplish this work, most of them are electronic devices the rest are lasers sources each has its specifications. Below are the devices with their specifications.

\subsubsection{Power Supply}

DC power supply used in this work for biasing the diode its output current range from $0-1.5 \mathrm{~A}$, and the voltage ranges from $0-15 \mathrm{~V}$ from supplied from LEYBOLD DEDACTIC GMBH, Germany [8].

\subsubsection{Variable Resistance}

A 5 ohms variable resistance used in this work was made in England by Philips Harris [9].

\subsubsection{Digital Multimeters}

A digital multimeter (DMM) is a test tool used to measure two or more electrical values - principally voltage (volts), current (amps) and resistance (ohms). Digital meter used in this work was made in China [10].

\subsubsection{Lasers}

\section{I-He-Ne Laser:}

$\mathrm{He}-\mathrm{Ne}$ laser is a type of gas lasers, the He-Ne used in this work is a type I class of lasers with output power less than one milli-watt, it operate with the fundamental mode 632.8 $\mathrm{nm}[11]$.

\section{II-Diode Laser:}

Diode laser is a semiconductor laser is a type II its output wavelength depends on the band gab energy. Semiconductor diode lasers are available at $(660 \mathrm{~nm}, 635 \mathrm{~nm}, 532 \mathrm{~nm}, 520$ $\mathrm{nm}, 445 \mathrm{~nm}, 405 \mathrm{~nm}$ ) wavelengths. The diode laser is most promising laser since its cheap and small [12]. The technology of the diode lasers now reaches the UV wavelengths. Diode laser used in this work was made china with wavelength of $660 \mathrm{~nm}$ and output power of less than 5 milli- watts [13].

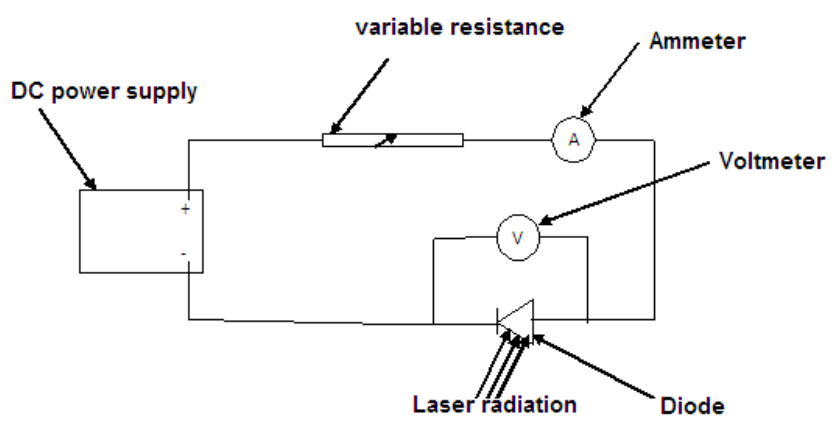

Figure 1. Schematic diagram of the experimental setup.

\subsection{Method}

The procedures to complete this works are done as follows in sequence:

a) The circuit was connected as shown in figure 1

b) First the characteristic of the diode was recorded.

c) Then the diode is subjected to the wavelength from the $\mathrm{He}$ - Ne laser for 5 minutes. And then the characteristic of the diode was recorded again.

d) The above step was repeated, with the total time of exposure of 10 minutes.

e) For the case of exposure of the diode to a laser radiation from $650 \mathrm{~nm}$ diode laser, a new diode is used and similar characteristics to that used with $\mathrm{He}-\mathrm{Ne}$ laser before the exposure were recorded.

f) The diode is subjected to the diode laser $650 \mathrm{~nm}$ for 5 minutes and then the Characteristics were recorded. 
g) The above step was repeated, with the total time of exposure of 10 minutes.

h) The IV characteristics of the diode before and after radiation with $\mathrm{He}-\mathrm{Ne}$ and diode lasers were plotted and compared.

\section{Results and Discussion}

The electrical characteristics of silicon diode before laser irradiation were recorded and tabulated in table 1 below:

Table 1. Electrical characteristics of diode before laser radiation.

\begin{tabular}{ll}
\hline $\mathbf{V} \pm \mathbf{0 . 0 1 V}$ & $\mathbf{I} \pm \mathbf{0 . 0 1} \mathbf{m A}$ \\
\hline 0 & 0 \\
0.1 & 0 \\
0.2 & 0 \\
0.3 & 0.01 \\
0.4 & 0.02 \\
0.42 & 0.03 \\
0.44 & 0.06 \\
0.45 & 0.08 \\
0.46 & 0.14 \\
0.47 & 0.18 \\
0.49 & 0.22 \\
0.51 & 0.24 \\
0.53 & 0.33 \\
\hline
\end{tabular}

The electrical characteristics of the diode at room temperature without subjected to laser radiation was plotted in figure 2 below:

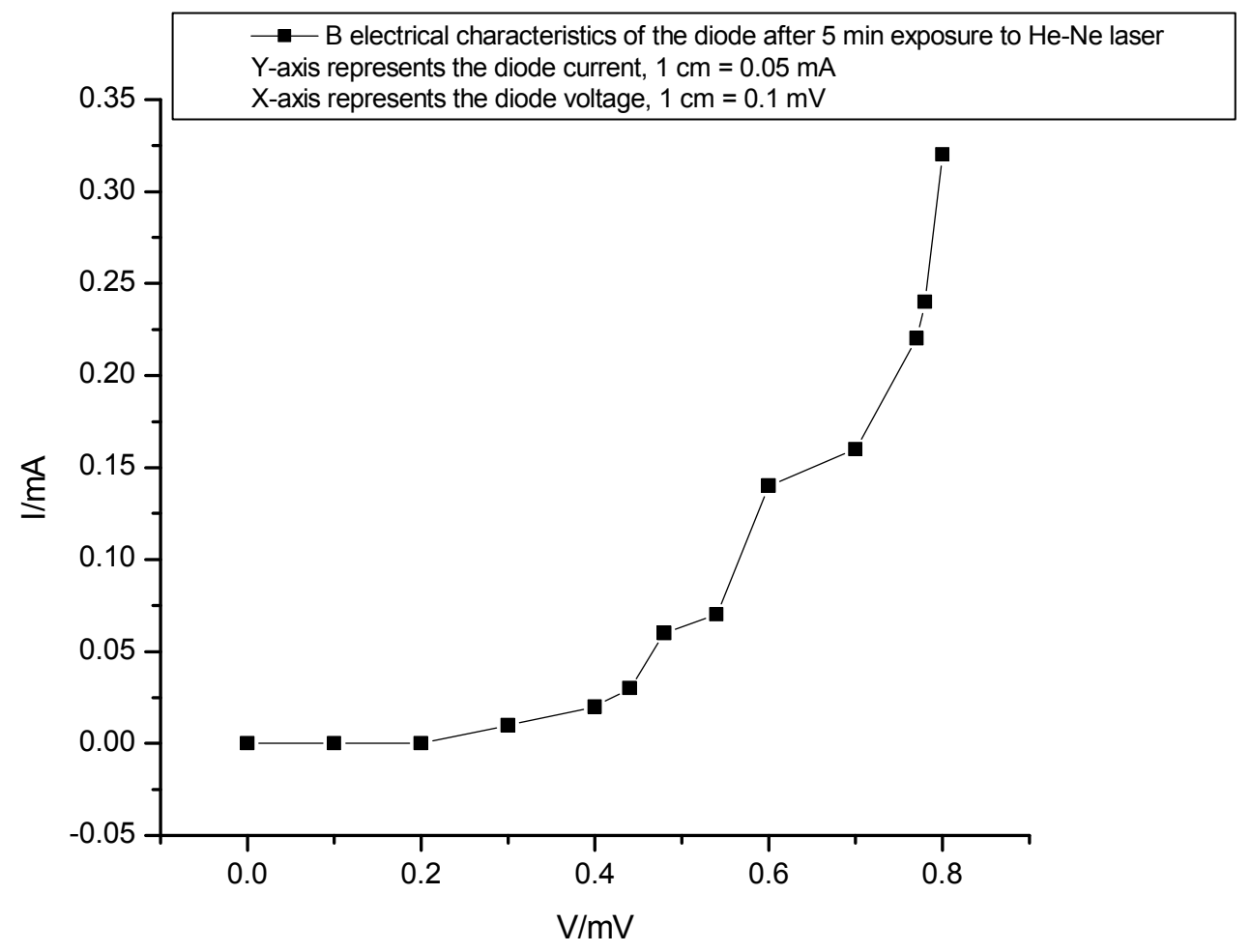

Figure 2. Electrical characteristics of the diode before lasers irradiations.

Figure 2 shows the expected electrical characteristics of the silicon diode but the obtained results showed a knee voltage smaller than should be for the silicon diode. From the relation figure 2 electrical resistance could be calculated by the inverse of slope of the tangent. Then the silicon diode was subjected to $\mathrm{He}-\mathrm{Ne}(632.8 \mathrm{~nm})$ laser radiation for five minutes and its electrical characteristics were recorded again, the obtained electrical characteristics of the silicon diode after Five minutes of He-Ne laser exposure were plotted in figure 3 . 


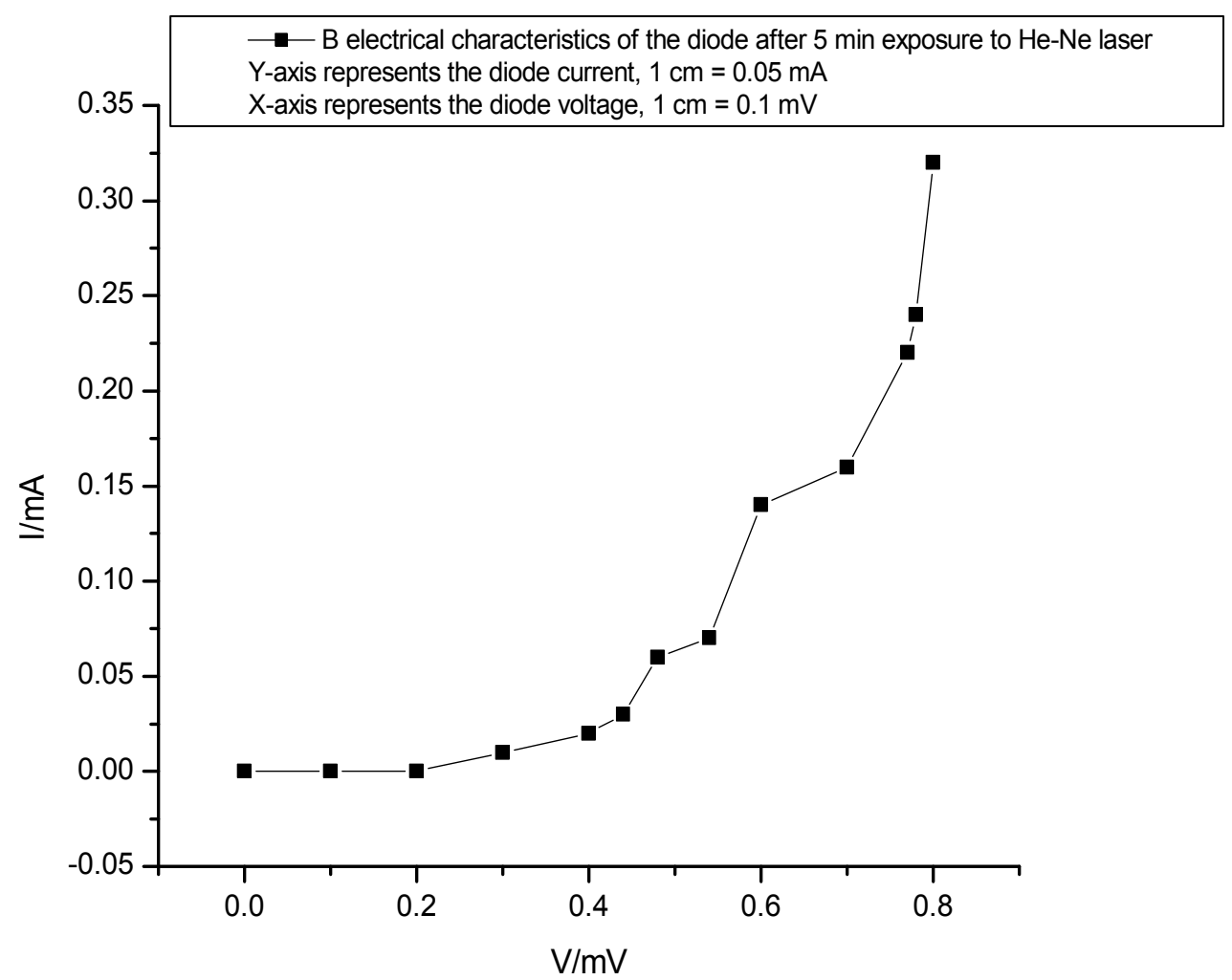

Figure 3. Electrical characteristics of the diode after 5 min. exposure to He-Ne laser.

Comparison of the electrical characteristics of the silicon diode before and after $\mathrm{He}-\mathrm{Ne}$ laser irradiation, figure 2 and figure 3 respectively appeared to be similar from first sight. To analyze the difference between the two characteristics and to have a reasonable results the diode is subjected again to a five minutes of the He-Ne radiation which means the diode is subjected to 10 minutes of the laser radiation then the electrical characteristics were recorded and together with the relations figures 2 and 3 plotted in figure 4 below.

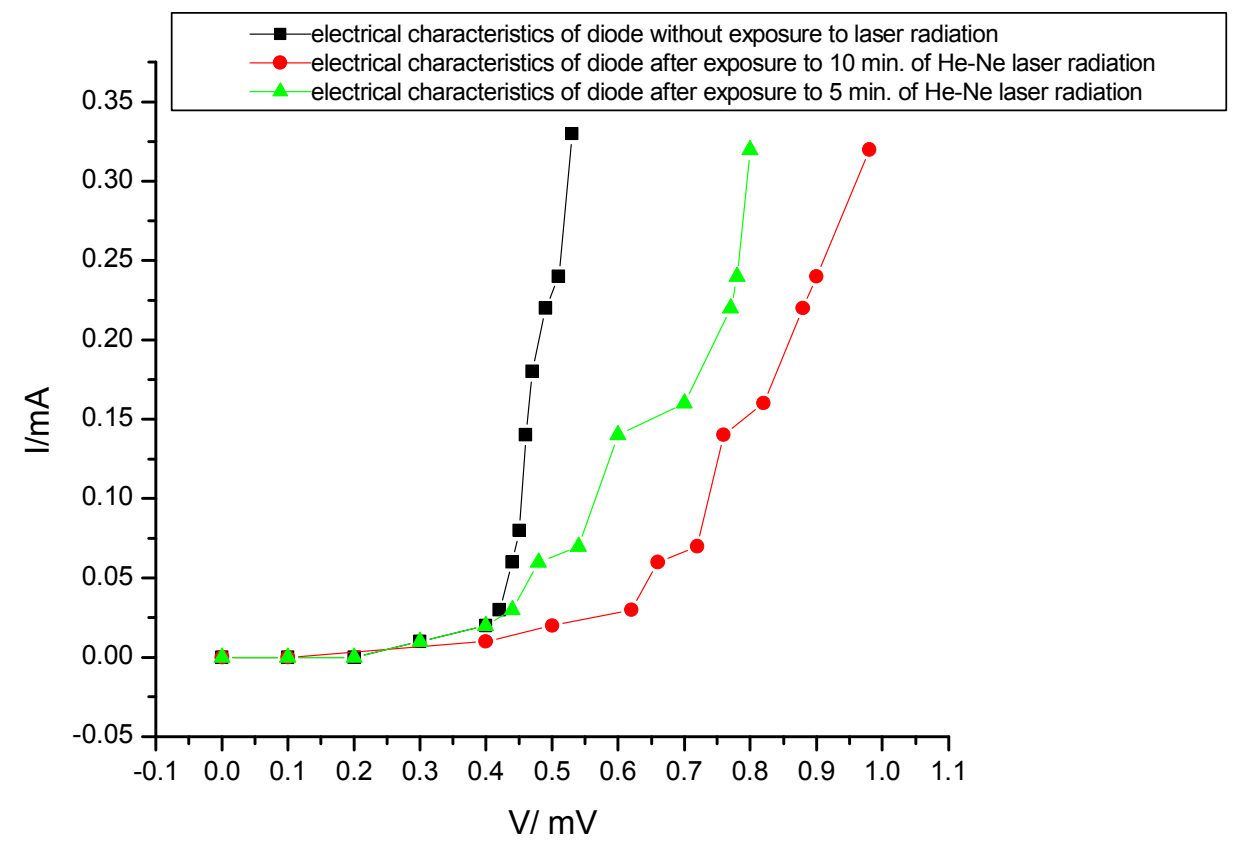

Figure 4. Electrical characteristics of diode with and without exposure to He-Ne laser radiation.

The applications of laser on the surface of the diode for different exposure times figure 4 showed that the effect of $\mathrm{He}-\mathrm{Ne}(632.8 \mathrm{~nm})$ laser radiation is a thermal effect, this is clear from the similarity between the obtained electrical characteristics of the diode before and after exposure to the $\mathrm{He}-\mathrm{Ne}$ laser when it compared with the electrical 
characteristics of the diode at different temperatures where at high temperature the characteristics shifts to the right in forward biasing. It should be noted here all the measurements are made for the forward biased condition only. Also figure 4 showed that when the time of exposure is increased the shifts in the diode electrical characteristics.

In the following section we describes the case of studying the effect of diode $(650 \mathrm{~nm})$ laser on the electrical characteristics of the silicon diode for different exposure times and compare the results with the results obtained with He-Ne laser:

The electrical characteristics of the diode after exposure to a five minutes of diode $650 \mathrm{~nm}$ laser radiation and to a ten minutes together with the electrical characteristics of the silicon diode before irradiations with diode laser $650 \mathrm{~nm}$ were plotted in figure 5 below.

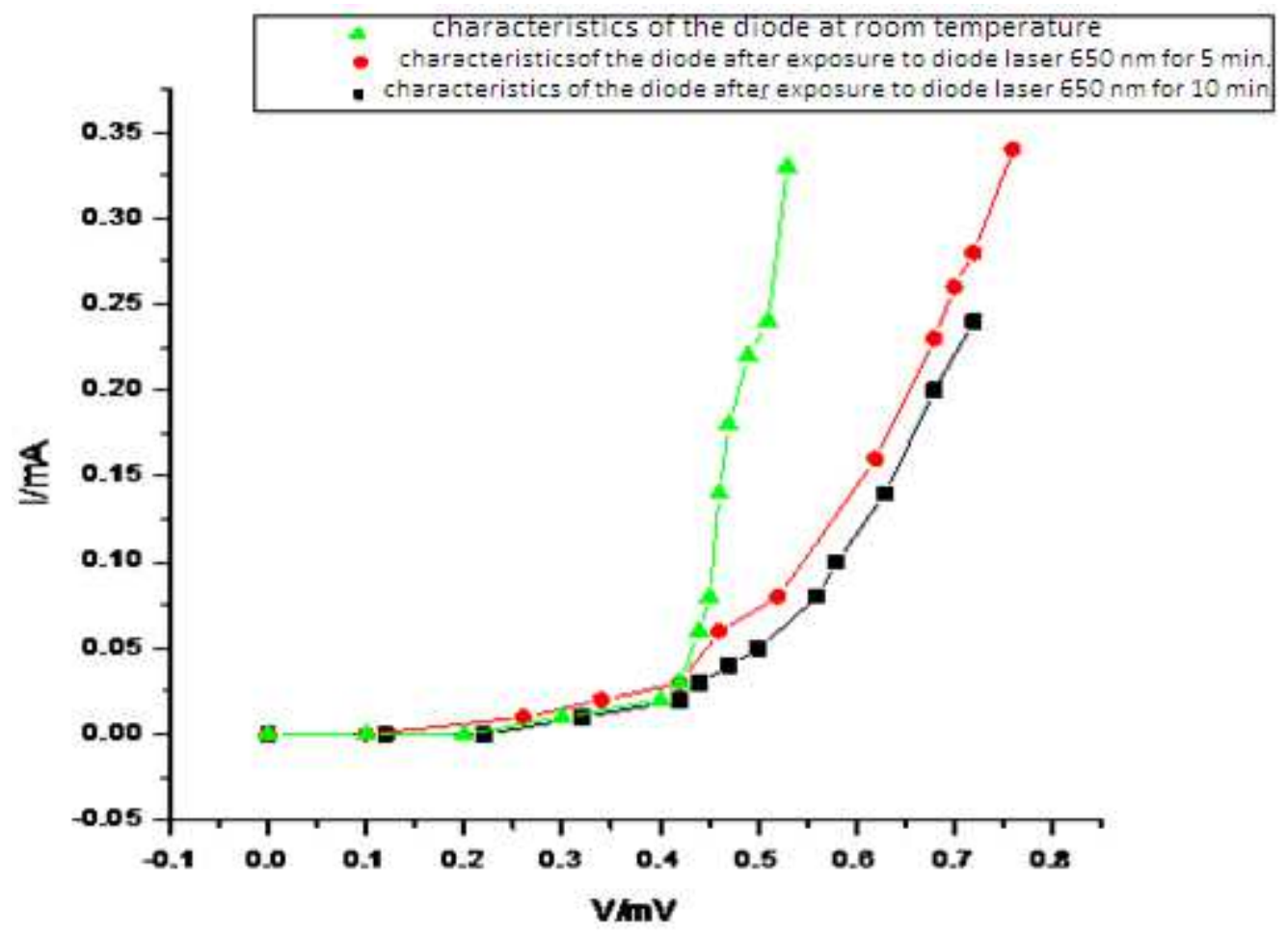

Figure 5. Electrical characteristics of diode without and with exposure to $650 \mathrm{~nm}$ diode laser radiation.

Figure 5 shows that the effect of the application of diode laser $650 \mathrm{~nm}$ on the surface of a silicon diode is similar to the effect of recording the characteristics at different temperature and this support the previous discussion of figure 4 .

When comparing between the electrical characteristics of the diode after exposure to $(632.8 \mathrm{~nm}) \mathrm{He}-\mathrm{Ne}$ laser and $(650$ $\mathrm{nm}$ ) diode laser it's clear that the exposure of the diode to a time of five minutes of the two radiations (at the middle of the three characteristics in figures 4 and 5 are comparable but for ten minutes the effect of He-Ne laser irradiation on the characteristics is different than that of the diode laser, this difference in the electrical characteristics of the same exposure time to $\mathrm{He}-\mathrm{Ne}$ and diode lasers is due to the facts that the two lasers has different properties such as power, wavelength, and beam diameter, etc. Future work will be on the investigations of these parameters on the electrical characteristics of silicon diode.

\section{Conclusions}

It concluded that:

The effect of He-Ne (632.8) and diode (650 nm) lasers with different exposure times on the electrical characteristics of silicon diode have been studied, their effects on the IV characteristics of the diode were recorded and compared to the electrical characteristics of the silicon diode before irradiation with two laser sources. It was found that the effect of laser irradiation on the electrical characteristics of silicon diode was similar to the effect of temperature; and thus the thermal mode of interaction is dominated in this application.

Also the effects from the two laser sources on the electrical characteristics were compared and it was shown that the effects of the He-Ne $(632.8 \mathrm{~nm})$ and diode $(650 \mathrm{~nm})$ were comparable for 5 minutes time of exposure but for exposure of ten minutes the effect of He-Ne laser irradiation on the electrical characteristics of the diode was different from that of the diode laser since the two laser sources has different properties.

\section{References}

[1] Frank J. Duarte, Tunable Laser Optics, Academic Press, 2003.

[2] John F. Ready, Industrial Applications of Lasers, Second Edition, Academic press, 1997. 
[3] Sheng S. Li, Semiconductor Physical Electronics, second edition, Springer, 2006.

[4] Charles A. Gross and Thaddeus A. Roppel, Fundamental of electrical engineering, CRC Press- 2012.

[5] Anthony J. De Maria and Thomas V. Hennessey, The $\mathrm{CO}_{2}$ Laser: The Workhorse of the Laser Material Processing Industry, 50th anniversary of laser devices, SPIE Professional Magazine, 2010.

[6] John C. Ion, Laser Processing of Engineering Materials, principles, procedure and industrial application, Elsevier Butterworth-Heinemann, London, 2005.

[7] Silicon Carbide diode SiC Final datasheets, 11/8/2017, $5^{\text {th }}$ Generation thin $\mathrm{Q} !^{\mathrm{TM}}$, Infineon, [online] available from: $\mathrm{http} / /$ www.infineon.com, visited October 2017.

[8] Instruction Sheet $52149 \mathrm{AC} / \mathrm{DC}$ power supply $0 \ldots 12 \mathrm{~V}$ - LD Didactic, [online] available from: http//www.lddidactic.de/documents/un-us/ga/ga/5/521/52149e.df,30/5/2017
[9] Variable resistance from Philips Harris, website: https://www.philipharris.co.uk/variable resistance, visited May 2017

[10] Digital millimeter, Fluke- china, available from: http://enus.fluke.com/training/training-

library/measurements/electricity/what-is-a-digitalmultimeter.html, visited 10/8/2017

[11] Phywe, Helium Neon Laser Products 8181-93 Info. 2007, https://www.phywe.de/en/geraetehierarchie/physics/modernphysics/quantum-physics/08181-93

[12] Diode laser, [online] from: https://en.wikipedia.org/wiki/Laser_diode, visited 9/6/2017

[13] Diode laser $650 \mathrm{~nm}$, product specifications, made by Dongguan Blue universe Laser Co., Ltd., China 2017. 